\title{
Starch-maleate-polyvinyl alcohol hydrogels with controllable swelling behaviors
}

\author{
Suh Cem Pang*, Suk Fun Chin, Soon Hiang Tay, Fui Mui Tchong \\ Department of Chemistry, Faculty of Resource Science and Technology, University of Malaysia Sarawak, 94300 Kota Samarahan, Sarawak, Malaysia
}

\section{A R T I C L E I N F O}

\section{Article history:}

Received 22 July 2010

Received in revised form

30 November 2010

Accepted 1 December 2010

Available online 8 December 2010

\section{Keywords:}

Native sago starch

Starch-maleate-PVA

Hydrogel

Swelling behavior

\begin{abstract}
A B S T R A C T
Starch-maleate-polyvinyl alcohol (SMP) hydrogels were prepared by reacting polyvinyl alcohol (PVA) with maleic acid (MA) substituted sago starch (SS). The substitution of MA and PVA onto the polysaccharide chain of sago starch was evidenced by the FTIR spectra which showed the presence of the carbonyl group absorption band of maleate ester, and increased intensity of the $\mathrm{C}-\mathrm{H}$ stretching absorption band. The surface morphology of SMP hydrogels as revealed by SEM micrograph was membrane-like with continuous matrices, and these samples were insoluble in both water and alkaline aqueous solution. TGA analysis showed that the SMP hydrogel exhibited higher thermal stability as compared to the RS, RPVA and SM samples. SMP hydrogel regenerated by freeze-drying showed substantially higher swelling ratio than hydrogel regenerated by direct precipitation under controlled conditions. The swelling behavior of SMP hydrogel could be easily controlled and modulated by varying the feeding composition of precursors or the regeneration methods. The high potential utility of SMP hydrogels in biomedical applications is envisaged by its biocompatibility, low toxicity, ease of preparation and low cost.
\end{abstract}

(c) 2011 Elsevier Ltd. All rights reserved.

\section{Introduction}

Hydrogels are hydrophilic polymeric materials that are capable of holding large amount of water or biological fluids in their three-dimensional networks. Hydrogels resemble living tissues in their elasticity, and they swell but do not dissolve when brought into contact with water or aqueous solutions (Alupei, Popa, Hamcerencu, \& Abadie, 2002). These unique properties make hydrogels useful materials for biomedical applications such as drug delivery systems, wound dressing, tissue engineering, soft contact lenses, protein sorption and recovery, and artificial implants (Bell \& Peppas, 1996; Gehrke, Vaid, \& McBride, 1998; Lee, Kung, \& Lee, 2005; Muzzarelli, 2009). Other practical applications of hydrogels include as flocculants for treatment of sludge, as well as for the release of agrochemicals, gas separation and removal of toxic heavy metal ions (Han, Chen, \& Hu, 2009; Karadag, Saraydin, Caldiran, \& Guven, 2000; Okazaki, Hamada, Fujii, Mizobe, \& Matsuzawa, 1995; Park \& Lee, 2001; Yin, Ju, Zhang, Wang, \& Yang, 2008).

Natural biopolymers are more favorable precursors for the preparation of hydrogels as they are nontoxic, biodegradable, renewable and abundant in nature ( $\mathrm{Li}, \mathrm{Xu}, \mathrm{Wang}$, Chen, \& Feng, 2009; Tang, Du, Hu, Shi, \& Kennedy, 2007). Among these natural biopolymers, starch has been shown to be a good precursor material for the preparation of hydrogels due to its great crosslinking ability in the presence of abundant hydroxyl $(\mathrm{OH})$ groups.

\footnotetext{
* Corresponding author. Tel.: +60 82 583017; fax: +60 82583160.

E-mail address: scpang@frst.unimas.my (S.C. Pang).
}

However, the hydrophilic nature of starch and its poor mechanical properties have hampered the development of unmodified starch as stable hydrogel (Fang, Fowler, Tomkinson, \& Hill, 2002; Swinkels, 1985; Zhang \& Sun, 2004). Hence, various biodegradable synthetic polymers such as poly(lactic acid) (PLA), poly(vinyl alcohol), (PVA) and poly(caprolactone), PCL have been blended together with the starch molecules in order to enhance its mechanical properties (Avella et al., 2000; Follain, Joly, Dole, \& Bliard, 2005). Among these polymers, PVA is one of the favorite choices due to its excellent compatibility with starch, ease of preparation, non-carcinogenic, inexpensive, biocompatible and biodegradable in nature.

However, blending of polymers may not produce desirable properties due to the poor adhesion between the polymers. Crosslinking is therefore necessary to improve the structural integrity of the polymer blends. Several cross-linkers that are well known for the cross-linking of starch include phosphoryl chloride (Woo \& Seib, 1997), epichlorohydrin, (Kuniak \& Marchessault, 2006), tri-sodium tri-metaphosphate (Gui-Jie, Peng, Xiang-Sheng, Xing, \& Tong, 2006) and glutaradehyde (El-Tahlawy, Venditti, \& Pawlak, 2007). However, these cross-linkers are harmful and toxic and therefore have limited their biomedical applications. In this study, we have attempted to use maleic acid (MA) as a cross-linker between starch and PVA through the esterification reaction in order to enhance the mechanical properties and swelling behaviors of the hydrogels formed. MA is being used as a type of food additive, and it is non-toxic and biodegradable in nature. It is therefore a favorable precursor for biomaterial synthesis. However, only few authors have reported the use of MA as a cross-linker with starch (Biswas, Shogren, Kim, \& Willett, 2006; Xing, Zhang, Ju, \& Yang, 